\title{
Why are children more resistant to mortality from severe infections?
}

\author{
Rose B Joachim ${ }^{1}$ \& Lester Kobzik*,1 \\ ${ }^{1}$ Department of Environmental Health, Harvard T.H. Chan School of Public Health, Boston, MA 02115, USA \\ *Author for correspondence: Ikobzik@hsph.harvard.edu
}

\section{'We all know it is 'good to be young', but what is the mechanism driving increased prepubertal survival in high-mortality infectious diseases?"}

First draft submitted: 3 August 2018; Accepted for publication: 20 August 2018; Published online: 13 November 2018

Keywords: estrogen $\bullet$ influenza $\bullet$ puberty $\bullet$ sepsis $\bullet$ severe infections

Inflammation is a balancing act - neither too much nor too little is desirable. Luckily, the host response can usually eradicate an infection while counterbalancing the inflammatory response with programs for resolution and repair. However in certain situations, this system of checks and balances breaks down. For example, in sepsis, dysregulation of the immune system can result in reactions ranging from inflammation-induced organ failure to dangerous immunosuppression [1]. The inability to return to homeostasis despite proper supportive care is also evident in the pathophysiology of other high mortality infectious diseases, suggesting that there might be an underlying mechanism connecting them.

Studies of individuals that are naturally resistant to infection-related mortality might help us better understand the mechanisms driving the underlying immune system dysregulation. Interestingly, human epidemiology indicates that children - especially prepubertal children - are one such group. For example, mortality data from the 1918 pandemic flu show that children between the ages of 5 and 14 (age of puberty onset then) had much better survival rates than young adults - despite similar rates of infection [2]. Importantly, this pattern extends to other high mortality infectious diseases such as tuberculosis, Ebola, yellow fever, pneumonia, chicken pox and sepsis (see Table $1[3]$.

Our laboratory has developed animal models of this intriguing phenomenon. We found that in comparison with postpubertal mice, prepubertal mice exhibited decreased morbidity and mortality in both $\mathrm{H} 1 \mathrm{~N} 1$ influenza infection and an endotoxemia model of sepsis $[4,5]$. Similar results have been reported in other mammalian models $[6,7]$. Our animal studies in particular highlight the importance of the pubertal transition in driving the survival difference. For example, survival from H1N1 influenza infection was higher in mice in which sexual development was halted via gonadectomy prior to puberty or by pharmacologic blockade using a gonadotropin-releasing hormone agonist [4].

\section{Why are prepubertal children more resilient?}

We all know it is 'good to be young', but what is the mechanism driving increased prepubertal survival in highmortality infectious diseases? We postulate that this resilience is the product of age-specific regulation of the immune system. Why might this age be immunologically special? First, in comparison with infants and young children, prepubertal children can manifest more competent immune responses. However, they have not yet experienced the sex-hormone mediated immunological reprogramming of puberty. Second, in the context of evolution, prepuberty is a unique life-stage characterized by specific energetic goals. At puberty, these goals shift dramatically from somatic maintenance (which includes immunity) to growth and reproduction and are subject to developmental plasticity. Taken together, we believe that studying the prepubertal developmental stage may provide insight into reversing an immune system dysregulation during high-mortality infectious diseases. 
The prepubertal immune system is at a unique place in development

Although innate immunity develops quickly throughout the first months of life, to ensure adequate protection against infection, infants rely heavily on immunoglobulins shared with them in their mothers' milk. Even after weaning, young children still lack an experienced adaptive immune system, leaving them susceptible to infectious diseases [8]. Entry into older childhood (by the age of 4 or 5) precipitates a drastic drop in this susceptibility. During this time, adaptive immunity matures, a diverse memory lymphocyte repertoire develops and the thymus steadily shrinks in size $[8,9]$.

What differentiates the immune system before and after puberty is a series of sexually dimorphic changes occurring in response to sex hormones. For example, at puberty the risk for autoimmune diseases increases in females while the risk for allergic diseases decreases in males [10,11]. In contrast with these sexually dimorphic changes, much less is known regarding the potential effects of gonadal steroids on both sexes. This is an important gap in knowledge considering that in the epidemiology of childhood resistance to mortality from severe infections and in data obtained from animal models, the age-related survival difference is similar in both males and females.

Data from our group's animal studies indicate that estrogen may unilaterally disrupt prepubertal resistance to mortality in both sexes. In $\mathrm{H} 1 \mathrm{~N} 1$ influenza infection, the survival benefit gained by preventing puberty through gonadectomy was lost when estrogen or testosterone was administered. However, dihydrotestosterone, which, unlike testosterone, cannot be converted to estrogen, did not reverse the survival benefit of gonadectomy. Furthermore, estrogen receptor antagonism with fulvestrant in both male and female pubertal mice resulted in improved survival [4]. In contrast with our findings that treatment of prepubertal animals with estrogen increases mortality from endotoxemia and $\mathrm{H} 1 \mathrm{~N} 1$ influenza, data does exist in adult rodents and humans that demonstrate a survival benefit of estrogen in critical illness and certain infectious diseases [12,13]. These discordant findings are consistent with the known complexity of sex hormones, including opposite effects depending on dose [12].

The potential for estrogen to have sex-independent effects on the immune system is logical, considering the essential role that it plays in the pubertal development of both sexes. At the beginning of male and female puberty, plasma levels of estrogen rise, stimulating the pubertal growth spurt. Although the increase in estrogen occurs earlier in girls than in boys, the bioactive hormone levels for growth in both sexes are equivalent when growth velocity peaks. Estrogen is also critical for promoting skeletal maturation and the cessation of longitudinal growth [14]. The importance of estrogen to both male and female puberty provides a biological precedent for its ability to alter resistance to high-mortality infectious diseases in both sexes.

\section{The time between childhood \& puberty has an evolutionary significance}

We believe that additional clues to the unique immunological status of prepubertal children can be identified through the lens of evolutionary life history theory. Evolutionary life history theory deals with an organism's strategic distribution of energetic resources toward growth, reproduction and somatic maintenance (which includes immunity). The theory predicts that natural selection will foster physiological mechanisms that allow for plasticity in the allocation of energy by linking specific life history stages to different energetic trade-offs.

In humans, the shift from early childhood to prepuberty is marked by several key processes: adrenarche (the initiation of adrenal androgen production), deceleration of growth and changes in adipose composition [15]. While the energetic prerogatives of prepuberty are somatic maintenance and a slow but steady rate of growth, during puberty, resources are shifted to fueling reproductive development and a rapid growth spurt [9]. It is possible that an increase in postpubertal mortality from infections is an accidental consequence of this shift in energetic priorities. The evolutionary importance of this transition is reflected in the great degree of plasticity available in the timing of puberty. Challenging environmental conditions like famine or infectious disease can halt or in some cases expedite sexual development [16].

In summary, the prepubertal developmental stage has specific energetic priorities that are subject to significant developmental plasticity in the transition through puberty. We hypothesize that these factors may contribute to an immunological profile that is unique to prepubertal individuals.

\section{Next steps in exploring prepubertal resistance}

We currently lack a detailed mechanism for prepubertal resilience. We hypothesize that it relates to a unique prepubertal immune profile characterized by immuno-competence, developmental plasticity and a lack of exposure to sex hormones. In the following section, we present some important next steps in the exploration of this hypothesis. 
A conservative, but still important next step is to explore the prepubertal resistance phenomenon using additional animal models of infectious disease. A particularly useful next step would be to study a larger mammal, such as the rabbit, which exhibits immune responses to infection that are more similar to those found in humans [17,18]. Additionally, comparing prepubertal resistance across multiple infectious diseases will help identify common regulatory processes at work.

After characterizing new models of prepubertal resistance, additional studies are warranted to dissect the unique regulatory environment that is driving prepubertal survival, including the role of sex hormones. One intriguing observation is that an adoptive transfer of prepubertal, but not postpubertal, peritoneal cells prior to an endotoxemia improved the survival of postpubertal recipient mice [5]. This suggests that the factors promoting survival were contained within the cells. Isolation of the causal cell would allow for detailed molecular profiling that could illuminate the mechanisms behind age-dependent responses to infection. Additional mechanistic studies using pharmacologic inhibition or genetic disruption of the estrogen receptor family and more exploration of other hormones (e.g., progesterone) would be useful. Assessing the epigenetic effects of estrogen at puberty may also be a useful next step [19]. Finally, the discussion of evolutionary perspectives above emphasized the potential influence of changing metabolic and energy requirements on the immune system. Direct comparison of immuno-metabolism in key leukocyte populations across the pubertal divide may provide valuable information in this regard [20].

\section{Conclusion}

Based on epidemiological data in humans and preliminary experimental data in mice, prepubertal animals survive better than postpubertal animals following challenge with high-mortality infectious disease illnesses which are deadly, thanks in large part to dysregulation of host inflammation. Important clues to mechanisms are offered in this age group's unique immunological stage of development. A deeper dive into this hypothesis may also lead to therapeutics for restoring balance in the immune system in severe infections.

\section{Financial \& competing interests disclosure}

The authors have no relevant affiliations or financial involvement with any organization or entity with a financial interest in or financial conflict with the subject matter or materials discussed in the manuscript. This includes employment, consultancies, honoraria, stock ownership or options, expert testimony, grants or patents received or pending, or royalties.

No writing assistance was utilized in the production of this manuscript.

\section{References}

1. Singer M, CS D, Seymour C et al. The third international consensus definitions for sepsis and septic shock (sepsis-3). JAMA 315(8), 801-810 (2016).

2. Ahmed R, Oldstone MBA, Palese P. Protective immunity and susceptibility to infectious diseases: lessons from the 1918 influenza pandemic. Nat. Immunol. 8, 1188 (2007).

3. Joachim RB, Altschuler GM, Hutchinson JN, Wong HR, Hide WA, Kobzik L. The relative resistance of children to sepsis mortality: from pathways to drug candidates. Mol. Syst. Biol. 14(5), e7998 (2018).

4. Suber F, Kobzik L. Childhood tolerance of severe influenza: a mortality analysis in mice. Am. J. Physiol. Cell. Mol. Physiol. 313(6), L1087-L1095 (2017).

5. Joachim R, Suber F, Kobzik L. Characterizing prepubertal resistance to death from endotoxemia. Sci. Rep. 7(1), 16541 (2017).

6. Huang SSH, Banner D, Degousee N et al. Differential pathological and immune responses in newly weaned ferrets are associated with a mild clinical outcome of pandemic 2009 H1N1 Infection. J. Virol. 86(24), 13187-13201 (2012).

7. Watson DW, Kim YB. Modification of host responses to bacterial endotoxins: I. specifity of pyrogenic tolerance and the role of hypersensitivity in pyrogenicity, lethality and skin reactivity. J. Exp. Med. 118(3), 425-446 (1963).

8. Simon AK, Hollander GA, McMichael A. Evolution of the immune system in humans from infancy to old age. Proc. Biol. Sci. 282(1821), 20143085 (2015).

9. McDade TW. Life history theory and the immune system: steps toward a human ecological immunology. Am. J. Phys. Anthropol. 122(37), 100-125 (2003).

10. Ngo ST, Steyn FJ, McCombe PA. Gender differences in autoimmune disease. Front. Neuroendocrinol. 35(3), 347-369 (2014).

11. Laffont S, Blanquart E, Guéry J-C. Sex differences in asthma: a key role of androgen-signaling in group 2 innate lymphoid cells. Front. Immunol. 8, 1069 (2017).

12. Klein SL, Flanagan KL. Sex differences in immune responses. Nat. Rev. Immunol. 16, 626 (2016). 
13. Kawasaki T, Chaudry IH. The effects of estrogen on various organs: therapeutic approach for sepsis, trauma and reperfusion injury. Part 1: central nervous system, lung and heart. J. Anesth. 26(6), 883-891 (2012).

14. Styne DM, Grumbach MM. Physiology and disorders of puberty. In: Williams Textbook of Endocrinology. Elsevier, Philadelphia, PA, 1074-1218 (2016).

15. Hochberg Z. Juvenility in the context of life history theory. Arch. Dis. Child. 93(6), 534-539 (2008).

16. Hochberg Z, Belsky J. Evo-devo of human adolescence: beyond disease models of early puberty. BMC Med. 11, 113 (2013).

17. Frevert CW, Matute-Bello G, Martin TR. Rabbit models of pneumonia, peritoneal sepsis and lung injury BT: chemokine protocols. In: Chemokine Protocols: Methods in Molecular Biology Proudfoot AEI, Wells TNC, Power CA (Eds), Humana Press, Totowa, NJ, USA, 319-330 (2000).

18. Strandberg KL, Rotschafer JH, Vetter SM, Buonpane RA, Kranz DM, Schlievert PM. Staphylococcal superantigens cause lethal pulmonary disease in rabbits. J. Infect. Dis. 202(11), 1690-1697 (2010).

19. Thompson EE, Nicodemus-Johnson J, Kim KW et al. Global DNA methylation changes spanning puberty are near predicted estrogen-responsive genes and enriched for genes involved in endocrine and immune processes. Clin. Epigenetics. 10, 62 (2018).

20. O’Neill LAJ, Kishton RJ, Rathmell J. A guide to immunometabolism for immunologists. Nat. Rev. Immunol. 16(9), 553-565 (2016). 